\title{
Lyapunov-Max-Plus-Algebra Stability in Predator-prey Systems Modeled with Timed Petri Net
}

\author{
Subiono $^{1}$ and Zumrotus Sya'diyah ${ }^{2}$
}

\begin{abstract}
In this paper, we discuss the notion of max-plus algebra and their properties. We also construct a model of predator-prey systems with timed Petri net and analyze the stabilization of the systems. Furthermore, we analyze the periodic behavior of the systems. Using the Lyapunov stability theory, we will obtain the sufficient condition for the stabilization problem and the periodic duration of the oscillation will be also determined.
\end{abstract}

Keywords—predator-prey systems, stability of the systems, Lyapunov method, max-plus algebra, timed petri net

Abstrak-Dalam paper ini, dibahas pengertian aljabar max-plus dan sifat-sifatnya. Selanjutnya dengan menggunakan Petri net berwaktu dikonstruksi suatu model sistem mangsa-pemangsa dan dianalisa kestabilan serta perilaku periodik sistem. Dengan menggunakan kestabilan Lyapunov diperoleh syarat cukup masalah kestabilan dan durasi osilasi dapat ditentukan.

Kata kunci-sistem mangsa-pemangsa, kestabilan sistem, kestabilan Lyapunov, aljabar max-plus, petri net berwaktu

\section{INTRODUCTION}

$\mathrm{G}$ enerally, the state of systems changes as time changes. The state spaces are expected to change at every tick of the clock. These kinds of systems are called time driven systems. In addition to this ones, there are some of them evolve in time by the occurrence of events at possible irregular time intervals, i.e. not necessarily coinciding with clock ticks. In this case, the state transition is a result of the other harmonic events. This kind of systems is called event driven systems [1].

Discrete event systems are defined by an event driven systems with discrete states. Discrete event systems are linear if they are formulated into max plus algebra. In this kind of systems, event is more decisive than time [2]. Transportation systems, manufacture process, and telecommunication network can be analyzed with discrete event systems [3].

Max plus algebra is the useful approach to represent the discrete event systems. This approaching makes us possible to determine and analyze various kinds of systems properties. Therefore, the model of these ones will be linear over max plus algebra. But in conventional algebra, it does not a linear. Because of the linearity, we can analyze the systems in max plus algebra easier and simpler than the conventional systems [3].

A Petri net is a mathematical modelling tool which can be applied to represent the state evolution of the discrete event systems. Petri net is called autonomous if every transition in this Petri net has at least an input place. This means that there is no transition which is enabled without any condition. In other words, autonomous Petri net does not have a transition which is always be enabled [4]. Timed Petri net is an extension of Petri net. Timed

Subiono is with Department of Mathematics, FMIPA, Institut Teknologi Sepuluh Nopember, Surabaya, 60111, Indonesia. E-mail: Subiono2008@matematika.its.ac.id.

Zumrotus Sya'diyah is Graduated Bchelor Degree of Department of Mathematics, FMIPA, Institut Teknologi Sepuluh Nopember, Surabaya, 60111, Indonesia. E-mail: zoe_zoe_40@yahoo.com.
Petri net is a Petri net with state changing time is considered. This state changing time is called holding times [3]. In this paper, we use the autonomous timed Petri net to model the predator-prey systems.

In the previous research, the predator-prey systems has modeled with timed Petri net. This research results a model of predator-prey systems which is consistent with the real predator-prey behavior in real life [5]. In this paper we will modify that one by adding some holding times, condition and event. This Model is inspired by the timed Petri net model of queuing systems with one server that discussed in [3].

There are five conditions and four events in this timed Petri net model of predator-prey systems. The conditions are preys in rest, preys do their activity, predators are idle, preys are in danger, and preys are being eaten. And, the events are preys finish their resting, preys at threat, predators start attacking preys and predators leave the preys. There are also five holding times related to each condition of the systems. It means that time which is spent by a condition to make an event take place is considered. This treatment is applied for each condition. We will analyze the stabilization and the dynamical behavior of the systems. Using the Lyapunov stability theory, we will obtain the sufficient condition for the stabilization problem and the periodic duration of the oscillation will be also determined. As a conclusion, we give some notes of the discussion and the future work.

\section{METHOD}

\section{A. Max-Plus Algebra and Some Related Notaion}

We will give a briefly introduction to max plus algebra that will be used in the next discussion, more detail explanation about max plus algebra can be found in [6]. The domain of max plus algebra is the set $\mathbb{R}_{\varepsilon}=$ $\mathbb{R} \cup\{\varepsilon=-\infty\}$ where $\mathbb{R}$ denotes the set of real number. The basic operations in max plus algebra are maximization (denoted by $\otimes$ ) and addition (denoted by $\otimes$ ). For $\mathrm{x}, \mathrm{y}$, $\in \mathbb{R}_{\varepsilon}$, we get:

$x \otimes y=\max \{x, y\}$ and $x \otimes y=x+y$ 
The set $\mathbb{R}_{\varepsilon}$ with operation maximization and addition will be written as $\mathbb{R}_{\max }$. For any $\mathrm{x} \in \mathbb{R}_{\varepsilon}$ satisfy the equation bellow,

$x \otimes \varepsilon=x=\varepsilon \otimes x$ and $x \otimes 0=x=0 \otimes x$

In the max plus algebra $\mathbb{R}_{\max }$, two operations, i.e. addition and multiplication, are denoted by $\otimes$ and $\otimes$ respectively. For two matrices $A$ and $B$ over $\mathbb{R}_{\max }^{m \times n}$, the addition of the matrices given by:

$[A \otimes B]_{i, j}=a_{i, j} \otimes b_{i, j}=\max \left\{a_{i, j}, b_{i, j}\right\}$

where $i=1,2,3, \ldots, m$ and $j=1,2,3, \ldots, n$. The multiplication of two matrices $A \in \mathbb{R}_{\max }^{m \times p}$ and $B \in \mathbb{R}_{\max }^{p \times n}$ is given by:

$[A \otimes B]_{i, j}=\bigoplus_{k=1}^{p}\left(a_{i, j} \otimes b_{i, j}\right)=\max \left\{a_{i, j}, b_{i, j}\right\}$

Where $i=1,2,3, \ldots, m$ and $j=1,2,3, \ldots, n$. There is an analogy between $\otimes$ and + and also $\otimes$ and $\times$. Therefore we choose the symbols $\otimes$ and $\otimes$. As in conventional algebra, an eigenvalue and a corresponding eigenvector of a square matrix of size $n \times n$ also exists in max-plus algebra, i.e. if we give the equation

$A \otimes x=\lambda \otimes x$

then the vector $\mathrm{x} \in \mathbb{R}_{\max }^{\mathrm{n}}$ and scalar $\lambda \in \mathbb{R}$ are respectively called an eigenvector and a corresponding eigenvalue of the matrix $\mathrm{A}$ with vector $\mathrm{x} \neq(\varepsilon, . ., \varepsilon)^{\mathrm{T}}$ where sign ${ }^{\mathrm{T}}$ represents transpose.

Let be given matrix $A \in \mathbb{R}_{\max }^{n \times n}$, a directed graph of matrix $A$ is denoted by $G(A)=(E, V)$. Graph $G(A)$ has $n$ nodes (vertices), the $s$ et of all nodes of graph $G(A)$ is denoted by $V$. An arc (edge) from node $j$ to $i$ exists if $a_{i, j}$ $\neq \varepsilon$, this arc is denoted by (j,i). The set of all arcs of graph $\mathrm{G}(\mathrm{A})$ is denoted by $\mathrm{E}$. The weight of $\operatorname{arc}(\mathrm{j}, \mathrm{i})$ is a value of $\mathrm{a}_{\mathrm{i}, \mathrm{j}}$, this one is denoted by $\mathrm{w}(\mathrm{j}, \mathrm{i})=\mathrm{a}_{\mathrm{i}, \mathrm{j}}$. If $\mathrm{a}_{\mathrm{i}, \mathrm{j}}=\varepsilon$ then $\operatorname{arc}$ $(\mathrm{j}, \mathrm{i})$ does not exist. A sequence of $\operatorname{arc}\left(\mathrm{i}_{1}, \mathrm{i}_{2}\right)\left(\mathrm{i}_{2}, \mathrm{i}_{3}\right), \ldots\left(\mathrm{i}_{\mathrm{l}-1}, \mathrm{i}_{1}\right)$ of a graph is called a path. A path is called elementer if its nodes has only one incoming and one outgoing arc. A circuit is a close elementer path, i.e.:

$\left(i_{1}, i_{2}\right)\left(i_{2}, i_{3}\right), \ldots,\left(i_{l-1}, i_{1}\right)$

The weight of a path : $p=\left(i_{1}, i_{2}\right)\left(i_{2}, i_{3}\right), \ldots,\left(i_{l-1}, i_{1}\right)$ is denoted by $|p|_{\mathrm{w}}$ with

$|p|_{\mathrm{w}}=\left(a_{i 2, \mathrm{i} 1}+a_{i 3, i 2}+\cdots+\mathrm{a}_{i l, i l-1}\right)$

The lenght of a path $\mathrm{p}$ is the sum of arc in the path $\mathrm{p}$ and it is denoted by $|p|_{1}$. The average weight of a path $p$ is the weight of path $p$ divided by the lenght of path $p$, i.e.

$\frac{|p|_{w}}{|p|_{l}}=\frac{\left(a_{i 2, i 1}+a_{i 3, i 2}+\cdots+a_{i l, i l-1}\right)}{l-1}$

Circuit mean is the average weight of a circuit which also acts as the eigenvalue of the matrix A. An algorithm to compute an eigenvalue and a corresponding eigenvector of a square matrix A can be found in [7]. This algorithm is an iterative algorithm of a linear equation of the form:

$\mathrm{X}(\mathrm{k}+1)=\mathrm{A} \otimes \mathrm{x}(\mathrm{k}) \cdot \mathrm{k} \geq 0$

The periodical property of the system is related to the cycle time vector

$\lim _{k \rightarrow \infty} \frac{x(k)}{k}$

For non negative number $M \geq 0$ let be a matrix $A_{m} \in \mathbb{R}_{\max }^{n \times n}$ with $0 \leq \mathrm{m} \leq \mathrm{M}$ and $x(m) \in \mathbb{R}_{\max }^{n}$ for $M \leq m$ $\leq-1$, then the difference equation of $M$ th-order will be written as follow:
$x(k)=\bigoplus_{m=0}^{M}\left(A_{m} \otimes x(k-m)\right), k \geq 0$

A difference equation of $M$ th-order with $\mathrm{A}_{0} \neq \varepsilon$ can be transformed into a difference equation of 1 st-order that is given by Equation 1, as follow:

$\widetilde{\mathrm{X}}(k+1)=\tilde{A} \otimes \widetilde{\mathrm{X}}(k), k \geq 0$,

where,

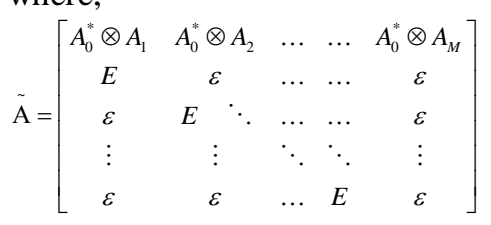

and

$A_{0}^{*}=\oplus_{i=0}^{n-1} A_{0}^{\otimes i}$

the matrix $E$ is identity matrix. The information about this equation can be found in [7]. The Equation 3 that satisfies 4 and 5 will be called as the autonomous equation.

\section{B. Petri Net and Timed Petri Net Theory}

We discuss about Petri net, timed Petri net and their theory related to the problem discussed in this paper. Petri net is a 4-tuple $(P, T, A, w)$, where $P$ is a finite set of places, i.e. $P=\left\{p_{1}, p_{2}, \ldots p_{m}\right\}, T$ is a finite set of transitions, i.e. $T=\left\{t_{1}, t_{2}, \ldots t_{n}\right\}, A$ is a set of arcs, i.e. $A \subseteq$ $(P \times T) \cup(T \times P)$ and $\mathrm{W}$ is weight function, i.e., $\mathrm{w}: \rightarrow\{1,2,3, \ldots\}$.

Petri net graph consist of two shapes. Ones are rectangles or lines and the others are circles. The rectangles and circles respectively represent the transition and the place. Mostly, a transition and a place can be respectively interpreted as an event and a condition such as an event can be occurred.

A Petri net marking vector is a vector of size $n \times 1$, where $n$ is the number of place in the petri net. This vector is denoted by:

$x=\left[\begin{array}{c}x\left(p_{1}\right) \\ x\left(p_{2}\right) \\ \vdots \\ x\left(p_{n}\right)\end{array}\right]$

where the $i$-th lement of this vector represents the number of token in $i$-th place $p_{i}$. Next, a transition $t_{j}$ is said to be enabled if $x\left(p_{i}\right) \geq w\left(p_{i}, t_{j}\right)$ for all $p_{i} \in I\left(t_{j}\right)$, where $I\left(t_{j}\right)=\left\{p_{i} \in P \mid\left(p_{i}, t_{j}\right) \in A\right\}$.

If a transition is enabled, then this transition can be fired. So, there is a token movement in the Petri net model of systems.

There are two kinds of representation matrices of a Petri net i.e., the backward and forward incidence matrices which are respectively denoted by $A_{b}$ and $A_{f}$. The elements of $A_{b}$ and $A_{f}$ are defined by:

$A_{b}\left(i, j \stackrel{\text { def }}{=} w\left(p_{i}, t_{j}\right)\right.$ and $A f(i, j) \stackrel{\text { def }}{=} w\left(t_{j}, p_{i}\right)$

where $i=1,2, \ldots m$ and $j=1,2, \ldots n$. From the definition of backward and forward incidence matrices, we can define an incidence matrix $A$ in term of $A_{b}$ and $A_{f}$ as follows:

$A=A_{b}-A_{f}$

So we get the new state after firing the enabled transition as follows:

$$
x^{\prime}\left(\left[p_{1}, p_{2}, \ldots p_{n}\right]^{T}\right)=x\left(\left[p_{1}, p_{2}, \ldots p_{n}\right]^{T}\right)+A e_{j}
$$

where $e_{j}$ represents the firing of $j$-th enabled transition.

A timed Petri net is a 6-tuple $\left(P, T, A, w, M_{0}, S\right)$ where $M_{0}$ is initial marking vector function, i.e. $M_{0}: P \rightarrow\{0,1,2,3, \ldots\}$ 
and $S$ is time structure related to all of the place in Petri net. This structure of time called holding time which means as the time a token have to spend in a place before contributing to the enabling of a transition. More discussion about Petri net is given in [8].

\section{RESULTS AND DISCUSSION}

Consider the equation of the first order difference equation of the systems that be given by:

$x(n+1)=f[n, x(n)], x\left(n_{0}\right)=x_{0}, n \in \mathbb{N}_{n_{0}}^{+}$

where $\mathbb{N}_{n_{0}}^{+}=\left\{n_{0}, n_{0}+1, \cdots, n_{0}+k, \cdots\right\}, n_{0} \geq 0$, $x(n) \in \mathbb{R}^{n}$ and $f: \mathbb{N}_{n_{0}}^{+} \times \mathbb{R}^{n} \rightarrow \mathbb{R}^{n}$ is a continuous function in $x(n)$. There is also a continuous function $\alpha:[0, \infty) \rightarrow:[0, \infty)$. If $\alpha(0)=0$ and $\alpha$ is an increasing function, then $\alpha \in \mathcal{K}$ where $\mathcal{K}$ is a class of function.

There is a Lyapunov function given by:

$v(n, x(n))$ with $v: \mathbb{N}_{n_{0}}^{+} \times \mathbb{R}^{n} \rightarrow \mathrm{R}_{+}^{p}$

And $\Delta v=(n+1, x(n+1))-v(n, x(n))$. So, we get the result which is given in the following theorem.

Let $(n,(n))$ with $v: \mathbb{N}_{n_{0}}^{+} \times \mathbb{R}^{n} \rightarrow \mathrm{R}_{+}^{p}$ be a continuous function in $x$ and define a function as follows:

$v_{o}(n, x(n))=\sum_{i=1}^{p} v_{i}(n, x(n))$

such that this equation satisfies the following conditions:

1. $b(|x|) \leq v_{0}(n, x(n)) \leq a(|x|), a, b \in K$

2. $\Delta v(n, x(n)) \mathrm{k} \leq \omega(n, x(n))$ for $n \in \mathbb{N}_{n_{0}}^{+}, x(n) \in \mathbb{R}^{n}$ where $\omega: \mathbb{N}_{n_{0}}^{+} \times \mathbb{R}_{+}^{p} \rightarrow \mathbb{R}^{p}$ is a continuous function and $\mathbb{N}$ is the set of natural number. It is assumed that for $h \in \mathbb{R}_{+}^{p}$ we have $g\left(n, h_{n}\right)=h_{n}+\omega\left(n, h_{n}\right)$ is a non decreasing function in $h$, with $0<\lambda<B$ are given, and $a(\lambda)<b B, \lambda, B \in[0, \infty)$. If $h(n+1)=g(n, h(n))$ with $h\left(n_{0}\right)=h_{0} \geq 0$ then system in (7) is stable. More information about this Theorem can be found in [9].

\section{A. Lyapunov Stabilization in Discrete Event Systems Modeled by Petri Net}

Let bet $e_{k}$ denotes the firing vector at time $k$, and $M_{k}=\left[M_{k}\left(p_{1}\right), M_{k}\left(p_{2}\right), \ldots M_{k}\left(p_{m}\right)^{T}\right.$ denotes the firing vector at time $k$. So, we get a matrix equation which shows the dynamical behavior of systems modeled by Petri net as follows: $M_{k+1}=M_{k}+A e_{k}$.

If we fire some of enabled transitions $d$ with the corresponding firing vectors are $e_{0,1, \ldots} e_{d-1}$ at time $k$. Then we get the following marking vector $M^{\prime}$

$M^{\prime}=M+A^{T} e$, with $e=\sum_{k=0}^{d-1} e_{k}$

Equation 8 shows that the marking vector $M^{\prime}$ can be reached from the other marking vector $M$.

The discrete event systems modeled by Petri net has the following state $M_{k}=\left[M_{k}\left(p_{1}\right), M_{k}\left(p_{2}\right), \ldots M_{k}\left(p_{m}\right)^{T}\right.$ where $\left|M_{k}\right|=\sqrt{\left(M_{k}\left(p_{1}\right)\right)^{2}+\left(M_{k}\left(p_{2}\right)\right)^{2}+\cdots+\left(M_{k}\left(p_{n}\right)\right)^{2}}$

From this state, we can find the Lyapunov function which satisfies the Theorem as follows:

$v\left(k, M_{k}\right)=M_{k}^{T} \Phi$

where $\Phi$ is a vector with the appropriate size with each element is positive number. About the proving of this function as the Lyapunov one can be found in [9]. Then, we get the following proposition.

\section{Proposition 1}

A Petri net is stable if there is $m$ strictly positive vector $\Phi$, such that,

$\Delta v=e^{T} A^{T} \Phi \leq 0$

Moreover, the Petri net which does not satisfy the proposition above will be observed whether this Petri net is stabilizable. The criteria of a Petri net called as a stabilizable is defined in definition bellow.

\section{Definition}

A Petri net is stabilizable if there exist a sequence firing vector $e$ such that the system in Equation (8) is bounded.

The definition above can be explained more clearly. In order to attain this purpose, we use the following proposition.

3. Proposition 2

A Petri net is stabilizable if there is a transition firing vector $e$, such that

$\Delta v=A e \leq 0$ where $e=\sum_{k=0}^{d-1} e_{k}$

The detail information of those definition and propositions is given in [9].

\section{B. Timed Petri Net Model and Its Analysis of Predator Prey Systems}

In this section, we derive a timed Petri net model of a predator-prey system. Then, we analyze its stability and find the periodic duration oscillation of this system.

It is assumed that the predator species only depend on a single prey species as its food supply, the prey has unlimited food supply, and that there is no threat to the pray other than the specific predator. The timed Petri net model for predator-prey system is given in Figure 1, where the notation of places and transitions are explained bellow:

$\mathrm{R}$ : preys are resting

A : preys are doing their activity

I : Predators are idle

D : Preys in danger

E : Preys are being eaten

f : Preys finished resting

t : preys are at threat

$\mathrm{s}$ : predators start attack preys

d : predator departs (leaves the prey because has fully satisfied)

$C_{1,2}, C_{3}, C_{4}$ and $C_{5}$ are respectively the holding times corresponding to place R, A, I, D and E, where $C_{i} \in[0, \infty)$, $i=1,2,3,4,5$ and $C_{1}>C_{3}$.

From Figure 1, we get the incidence matrix bellow:

$$
A=A_{f}-A_{b}=\left[\begin{array}{llll}
1 & 0 & 0 & 0 \\
1 & 0 & 0 & 0 \\
0 & 0 & 0 & 1 \\
0 & 1 & 0 & 0 \\
0 & 0 & 1 & 0
\end{array}\right]-\left[\begin{array}{llll}
1 & 0 & 0 & 0 \\
0 & 1 & 0 & 0 \\
0 & 1 & 0 & 0 \\
0 & 0 & 1 & 0 \\
0 & 0 & 0 & 1
\end{array}\right]=\left[\begin{array}{cccc}
0 & 0 & 0 & 0 \\
1 & -1 & 0 & 0 \\
0 & -1 & 0 & 1 \\
0 & 1 & -1 & 0 \\
0 & 0 & 1 & -1
\end{array}\right]
$$

Furthermore, we have to find the vector $\Phi$ such that this vector satisfies the condition in Proposition 1. Because each element of $e^{T}$ is non negative, it is enough to show that

$A^{T} \Phi \leq 0$

where 0 is a vector with the appropriate size which all of its elements are zero. So, we get a vector: 
$\Phi=\left[\begin{array}{l}a \\ 0 \\ b \\ b \\ b\end{array}\right]$

such that:

$A^{T} \Phi=0$

According to Proposition 1, vector $\Phi$ given in (9) should be strictly positive. It is shown that the system modeled by timed Petri net above is not stable. But the system is stabilizable. It can be proved using Proposition 2. This stabilizable properties can be reached by solving the homogenous linear Equation below:

$A e=0$, where $e=\sum_{k=0}^{d-1} e_{k}$

Then we get

$e=\left[\begin{array}{l}y \\ y \\ y\end{array}\right]$

with $y \in \mathbb{N}$. Based on this vector, we can say that the system we discussed is stabilizable.

Now, we will find the oscillation periodic duration of the system. First, we have to find the recurrence equation in the max plus algebra.

From the model of timed Petri net in Figure 1, we can derive the equation of the system as follows:

$x_{1}(k)=C_{1} \otimes x_{1}(k-1)$

$x_{2}(k)=C_{2} \otimes x_{1}(k) \oplus C_{3} \otimes x_{4}(k-1)$

$x_{3}(k)=C_{4} \otimes x_{2}(k)$

$x_{4}(k)=C_{5} \otimes x_{3}(k)$

where $C_{i} \in[0, \infty), i=1,2,3,4,5, \quad x_{1}(k), x_{2}(k), x_{3}(\mathrm{k})$ and $x_{4}(k)$ respectively denotes the time of preys finish their resting at period $k$, the time of preys are threatened at period $k$, the time of predator starts attack the preys at period $k$, and the time of predator departs at period $k$.

These equations can be formed into matrices equation below:

$$
x(k)=A_{0} \otimes x(k) \oplus A_{1} \otimes x(k-1)
$$

where,

$$
\begin{aligned}
& x(k)=\left[\begin{array}{l}
x_{1}(k) \\
x_{2}(k) \\
x_{3}(k) \\
x_{4}(k)
\end{array}\right], \quad A_{0}=\left[\begin{array}{cccc}
\varepsilon & \varepsilon & \varepsilon & \varepsilon \\
C_{2} & \varepsilon & \varepsilon & \varepsilon \\
\varepsilon & C_{4} & \varepsilon & \varepsilon \\
\varepsilon & \varepsilon & C_{5} & \varepsilon
\end{array}\right] \text { and } \\
& A_{1}=\left[\begin{array}{cccc}
C_{1} & \varepsilon & \varepsilon & \varepsilon \\
\varepsilon & \varepsilon & \varepsilon & C_{3} \\
\varepsilon & \varepsilon & \varepsilon & \varepsilon \\
\varepsilon & \varepsilon & \varepsilon & \varepsilon
\end{array}\right]
\end{aligned}
$$

From Equation 10, we can determine matrix $A_{0}^{*}$ as follows:

$$
\begin{aligned}
A_{0}^{*} & =\oplus_{i=0}^{3} A_{0}^{\otimes i} \\
& =\left[\begin{array}{cccc}
\varepsilon & \varepsilon & \varepsilon & \varepsilon \\
C_{2} & \varepsilon & \varepsilon & \varepsilon \\
\varepsilon & C_{4} & \varepsilon & \varepsilon \\
\varepsilon & \varepsilon & C_{5} & \varepsilon
\end{array}\right]^{\otimes 0} \oplus\left[\begin{array}{cccc}
\varepsilon & \varepsilon & \varepsilon & \varepsilon \\
C_{2} & \varepsilon & \varepsilon & \varepsilon \\
\varepsilon & C_{4} & \varepsilon & \varepsilon \\
\varepsilon & \varepsilon & C_{5} & \varepsilon
\end{array}\right]^{\otimes 1} \\
& \left.\oplus\left[\begin{array}{cccc}
\varepsilon & \varepsilon & \varepsilon & \varepsilon \\
C_{2} & \varepsilon & \varepsilon & \varepsilon \\
\varepsilon & C_{4} & \varepsilon & \varepsilon \\
\varepsilon & \varepsilon & C_{5} & \varepsilon
\end{array}\right]^{\otimes 2} \quad{ }^{\varepsilon} \quad \begin{array}{cccc}
\varepsilon & \varepsilon & \varepsilon \\
C_{2} & \varepsilon & \varepsilon & \varepsilon \\
\varepsilon & C_{4} & \varepsilon & \varepsilon \\
\varepsilon & \varepsilon & C_{5} & \varepsilon
\end{array}\right] \\
& =\left[\begin{array}{ccccc}
0 & \varepsilon & \varepsilon & \varepsilon \\
C_{2} & 0 & \varepsilon & \varepsilon \\
C_{2}+C_{4} & C_{4} & 0 & \varepsilon \\
C_{2}+C_{4}+C_{5} & C_{4}+C_{5} & C_{5} & 0
\end{array}\right]
\end{aligned}
$$

Next, the standard autonomous equation of the system modeled by timed Petri net is given by:

$\tilde{x}(k+1)=\tilde{A} \otimes \tilde{x}(k)$

where $\tilde{x}(k)=\left[\tilde{x}_{1}(k), \tilde{x}_{2}(k), \tilde{x}_{3}(k), \tilde{x}_{4}(k)\right]^{T}$ and

$\tilde{\mathrm{A}}=A_{0}^{*} \otimes A_{1}=\left[\begin{array}{cccc}C_{1} & \varepsilon & \varepsilon & \varepsilon \\ C_{1}+C_{2} & \varepsilon & \varepsilon & C_{3} \\ C_{1}+C_{2}+C_{4} & \varepsilon & \varepsilon & C_{3}+C_{4} \\ C_{1}+C_{2}+C_{4}+C_{5} & \varepsilon & \varepsilon & C_{3}+C_{4}+C_{5}\end{array}\right]$

The next step to find the oscillation periodic duration of the system by calculating the eigen value of the matrix $\tilde{A}$. The graph of the matrix $\tilde{A}$ is shown in Figure 2,

From Figure 2, we g_et the eigenvalue as follows:

$\lambda=\max \left\{\frac{|p|_{w}}{|p|_{l}}\right\}=\max \left\{C_{1}, C_{3}+C_{4}+C_{5}\right\}$.

This result shows that the oscillation periodic duration of the system depends on $C_{1}$ and $C_{3,4}, C_{5}$. We noted that $C_{1}$ $>C_{3}$ so the predator has enough time to eat the prey. This is relevant with the real life.

Our next step is giving a value to each holding times. Let $C_{1}=6, C_{2}=2, C_{3}=4, C_{4}=1$ and $C_{5}=1$

be the holding times of the system. So, the eigen value of the matrix $\tilde{A}$ is given by,

$\lambda=\max \left\{C_{1}, C_{3}+C_{4}+C_{5}\right\}=\max \{6,6\}=6$.

This result shows that the oscillation periodic duration of the system is equal to 6 . This result must be different if we choose the other different holding times values.

\section{CONCLUSION}

In this section we give some notes of discussing especially in predator-prey system modeled by timed Petri net. The modified model given by:

$\left[\begin{array}{l}x_{1}(k) \\ x_{2}(k) \\ x_{3}(k) \\ x_{4}(k)\end{array}\right]=\left[\begin{array}{cccc}\varepsilon & \varepsilon & \varepsilon & \varepsilon \\ C_{2} & \varepsilon & \varepsilon & \varepsilon \\ \varepsilon & C_{4} & \varepsilon & \varepsilon \\ \varepsilon & \varepsilon & C_{5} & \varepsilon\end{array}\right] \otimes\left[\begin{array}{l}x_{1}(k) \\ x_{2}(k) \\ x_{3}(k) \\ x_{4}(k)\end{array}\right]$
$\oplus\left[\begin{array}{llll}C_{1} & \varepsilon & \varepsilon & \varepsilon \\ \varepsilon & \varepsilon & \varepsilon & C_{3} \\ \varepsilon & \varepsilon & \varepsilon & \varepsilon \\ \varepsilon & \varepsilon & \varepsilon & \varepsilon\end{array}\right] \otimes\left[\begin{array}{l}x_{1}(k-1) \\ x_{2}(k-1) \\ x_{3}(k-1) \\ x_{4}(k-1)\end{array}\right]$.

Next we transform the model to get a new model that is given by:

$$
\begin{aligned}
& \tilde{x}(k+1)=\tilde{\mathrm{A}} \otimes \tilde{x}(k) \\
& \text { where } \tilde{x}(k)=\left[\tilde{x}_{1}(k), \tilde{x}_{2}(k), \tilde{x}_{3}(k), \tilde{x}_{4}(k)\right]^{T} \text { and } \\
& \tilde{\mathrm{A}}=A_{0}^{*} \otimes A_{1}=\left[\begin{array}{cccc}
C_{1} & \varepsilon & \varepsilon & \varepsilon \\
C_{1}+C_{2} & \varepsilon & \varepsilon & C_{3} \\
C_{1}+C_{2}+C_{4} & \varepsilon & \varepsilon & C_{3}+C_{4} \\
C_{1}+C_{2}+C_{4}+C_{5} & \varepsilon & \varepsilon & C_{3}+C_{4}+C_{5}
\end{array}\right] .
\end{aligned}
$$

Then, we get the eigenvalue of $\tilde{A}$ as follows: $\lambda=\max \left\{\frac{|p|_{w}}{|p|_{l}}\right\}=\max \left\{C_{1}, C_{3}+C_{4}+C_{5}\right\}$. 
As we knew that the value of $\lambda$ is the oscillation periodic duration of the system. So we can conclude that this oscillation periodic duration of the system depends on $C_{1}$ and $C_{3}, C_{4}, C_{5}$. In order to the predator has enough time to eat the prey this could be $C_{1}>C_{3}$. Let, $C_{1}=6$, $C_{2}=2, C_{3}=4, C_{4}=1$ and $C_{5}=1$ be the holding times of the timed Petri net. So, we get the eigenvalue of the matrix $\tilde{A}$ as follows:

$\lambda=\max \left\{C_{1}, C_{3}+C_{4}+C_{5}\right\}=\max \{6,6\}=6$

According to this result, we conclude that the oscillation periodic duration of the system is equal to 6 . This result also shows that the holding time $C_{2}$ which is corresponding to place $A$ in Figure 2 does not play any role in determining the oscillation periodic duration of the system.

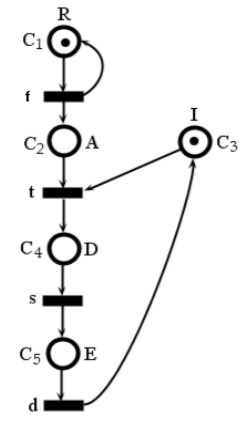

Figure 1. Timed Petri net model of predator-prey systems

\section{REFERENCES}

[1] I. Necoara, Model predictive control for max-plus-linear and piecewise affine systems, Neteherland, Technise Universiteit Delft, 2006.

[2] Subiono, The existence of eigenvalues for reducible matrices in max-plus algebra, Surabaya, Mathematics Department FMIPAITS, 2008.

[3] Subiono, Aljabar max plus dan terapannya. Surabaya, Department of Mathematic, ITS, 2010

[4] F. Bacelli, G. Cohen, G. Olsder, and J. Quadrat, Synchronization and Linearty, An algebra for discrete event system, Web Ed, 2001.

[5] Z. Retchkiman, "A Mixed lyapunov-max-plus algebra approach to the stability problem for a two species
For the future work, the discussing can be continued for finding out whether it is possible to construct a timed Petri net which represents the predator-prey system with all of the holding times in each place plays role in determining the oscillation periodic duration of the system such that the model more realistic than the previous one. For more advance studying, the holding times can be determinded as the interval values.

\section{ACKNOWLEDGEMENT}

We wish to give our gratitude to Prof. Zvi Retchkimann Konisberg for giving us the information about this project by his papers. We also really appreciate in every email that he sent related to this project.

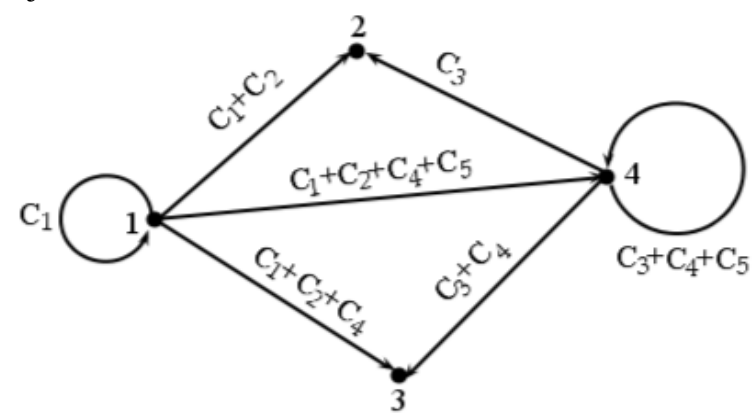

Figure 2. The graph of matrix $\tilde{A}$

ecosystem modeled with timed petri nets," International Mathematical Forums, vol. 5, pp. 1393-1408, 2010.

[6] Subiono and J. V. D. Woude, "Power algorithms for (Max,+)- and bipartite (Min, Max,+)-systems", Discrete Event Dynamic Systems: Theory and Applications, vol. 10, no. 4, pp, :369-389, 2000 .

[7] Subiono and N. Sofiyana, "Using max-plus algebra in the flow shop scheduling", IPTEK, The Journal for Technology and Science, vol. 20, 2009, pp. 83-87.

[8] D. Adzkiya, "Membangun model petri net lampu lalu lintas dan simulasinya," Tesis M.Si., Institut Teknologi Sepuluh Nopember. Surabaya, Indonesia, 2008.

[9] Z. Retchkiman, "The stability problem for discrete event dynamical systems modeled with timed petri nets using a lyapunov-max-plus algebra approach," International Mathematical Forum, vol. 6, pp. 541-566. 